Case Report

\title{
Histological Characteristics of Myxoid (Metaplastic) Meningioma in a 44-Year-Old Woman
}

\author{
Akiko Marutani $\mathbb{D}^{1},{ }^{1}$ Ryou Nakano, ${ }^{1}$ Noriyuki Nishi, ${ }^{2}$ and Tomonori Yamada ${ }^{1}$ \\ ${ }^{1}$ Department of Neurosurgery, National Hospital Organization, Osaka Minami Medical Center, Osaka, Japan \\ ${ }^{2}$ Oshima Medical Association Hospital, Kagoshima, Japan \\ Correspondence should be addressed to Akiko Marutani; akiko@naramed-u.ac.jp
}

Received 3 June 2019; Revised 10 September 2019; Accepted 26 September 2019; Published 6 December 2019

Academic Editor: Raffaele Palmirotta

Copyright (C) 2019 Akiko Marutani et al. This is an open access article distributed under the Creative Commons Attribution License, which permits unrestricted use, distribution, and reproduction in any medium, provided the original work is properly cited.

\begin{abstract}
Myxoid (metaplastic) meningioma is the rarest WHO grade 1 meningioma, and its histological characteristics are useful in diagnostics. We present the case report of a myxoid (metaplastic) meningioma in a 44-year-old woman to highlight the important histological features and observations that are critical for making an accurate diagnosis. We report a rare myxoid meningioma using magnetic resonance imaging (MRI) images and its histopathological features.
\end{abstract}

\section{Introduction}

Meningiomas are tumors that develop from arachnoid cells; approximately 15 histological subtypes with various characteristics are known. Myxoid meningioma is an extremely rare subtype of WHO grade I benign meningioma; only eight cases have been reported [1]. Here, we report a case of myxoid (metaplastic) meningioma using MRI images and its histopathological features.

\section{Case Report}

A 44-year-old woman presented with a headache and visual impairment in August 2017 and visited a local doctor one month later. A head computed tomography (CT) scan showed a $90 \times 90 \mathrm{~mm}$ mass in the right frontal lobe (Figure 1), and she was referred to our hospital. Head MRI revealed a mass presenting homogeneous hypointense signals on T1 images (Figure 2(a)) and hyperintense signals with a clear border on T2 images (Figure 2(b)) at the same site. Gadolinium- (Gd-) based contrast-enhanced MRI significantly enhanced the signals (Figure 3 ). On cerebral angiography, the mass had a sunburst appearance and refluxed from the middle meningeal artery (MMA). Based on these results, we diagnosed the mass a convexity meningioma and performed a craniotomy and tumorectomy.
Intraoperative findings showed that the tumor was hemorrhagic, dark red, and relatively soft and was partially accompanied by fibrous tissue (Figure 4(a)). After the MMA was treated, internal decompression was performed with Sonopet (Stryker Japan KK) to achieve total resection of the tumor, including removal of the infiltrated dura (Simpson grade I) (Figure 4(b)).

Histopathological analysis showed that fibrous connective tissue containing numerous blood vessels was subfractioned into leaflet-like portions (Figure 5(a)). The areas inside the leaflets were stained with Alcian blue and contained a mucoid matrix (Figure 5(b)). Immunostaining revealed that the tumor was positive for vimentin and epithelial membrane antigen (EMA) and negative for glial fibrillary acidic protein (GFAP). A small percentage (4.4\%) of tumor cells were positive for $\mathrm{Ki}-67$, and there was no finding of malignancy. Based on the above findings, we diagnosed the tumor as myxoid meningioma.

After surgery, the neurologic deficits resolved and the patient's progress was favorable. The patient was discharged two weeks after surgery and was able to walk independently upon discharge. Two years after surgery, recurrence had not been observed.

Written informed consent was obtained from the patient for publication of this case report. A copy of the written consent is available for review upon request. 


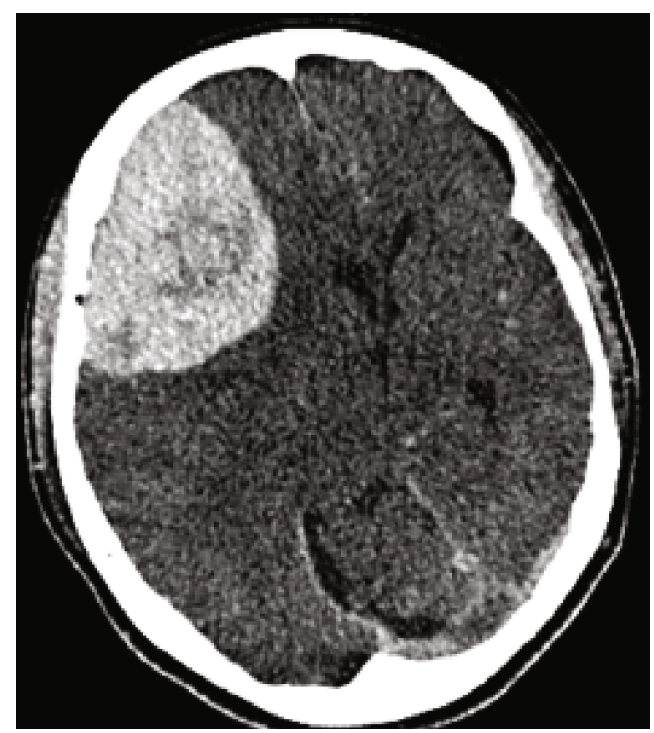

FIgURE 1: A head computed tomography $(\mathrm{CT})$ scan showed a $90 \times 90 \mathrm{~mm}$ mass in the right frontal lobe.

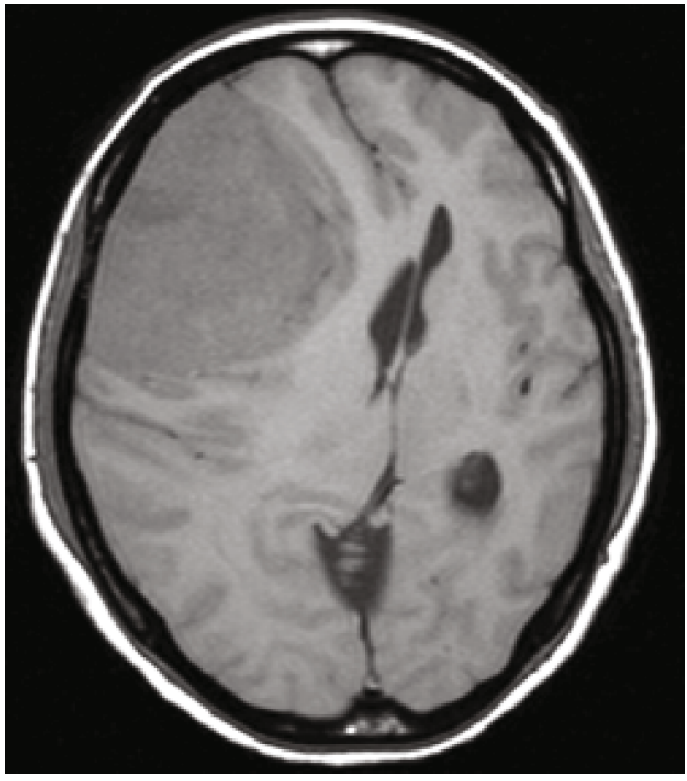

(a)

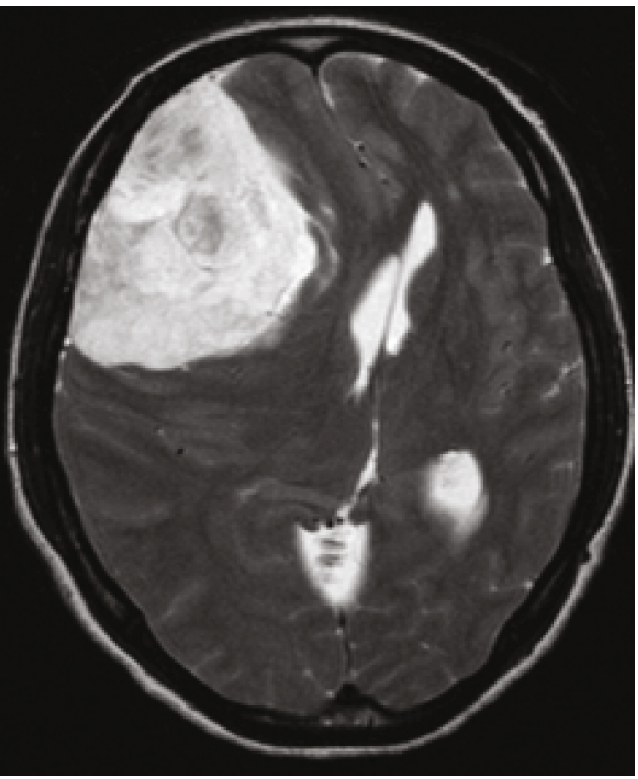

(b)

FIgURE 2: (a) Head magnetic resonance imaging (MRI) revealed a mass presenting homogeneous hypointense signals on T1 images. (b) At the same site, hyperintense signals with a clear border on T2 images.

\section{Discussion}

Metaplastic meningioma is a subtype of tumor in which a metaplasia of cells into stromal cells occurs locally to form bone, cartilage, lipids, and mucus. It includes osseous, cartilaginous, lipomatous, myxoid, xanthomatous, and melanin meningiomas [2]. Till now, most of reports of the metaplastic meningioma were osseous, lipomatous, xanthomatous, and smooth muscle meningiomas [3].

Myxoid meningioma is a rare type of metaplastic meningioma; pathological and immunohistological diagno- ses are important for definitive diagnosis and discrimination from other histological subtypes of meningioma and mucoid tumors (subtypes include osteosarcoma, chondrosarcoma, liposarcoma, chordoma, and xanthoma) [1]. In addition to whorl formation, psammoma body, and syncytial formation that are histopathological characteristics of meningioma, myxoid meningioma is characterized by an abundant mucoid matrix in the cytoplasm and large amounts of acidic mucopolysaccharides that stain with Alcian blue [4]. Our case is stained with Alcian blue and contained a mucoid matrix. 


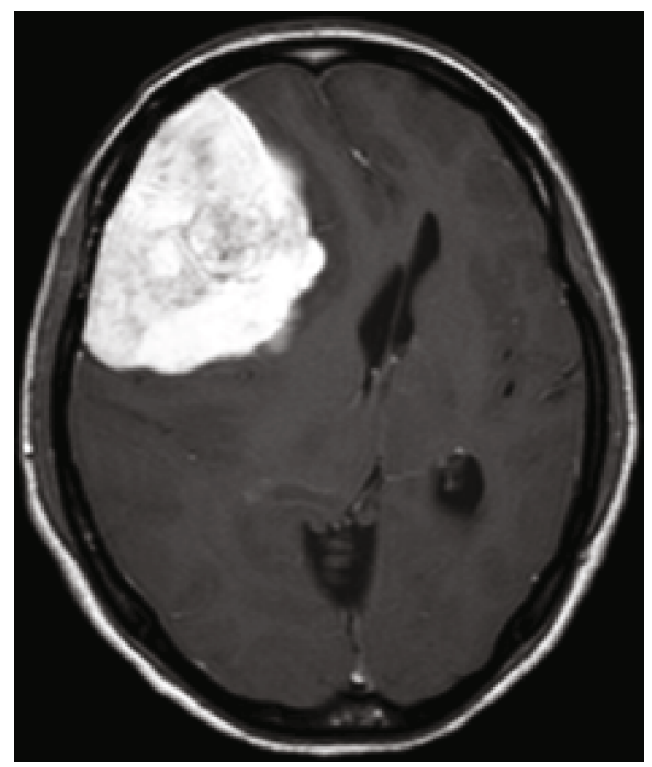

FIgURE 3: Gadolinium- (Gd-) based contrast-enhanced MRI significantly enhanced the signals.

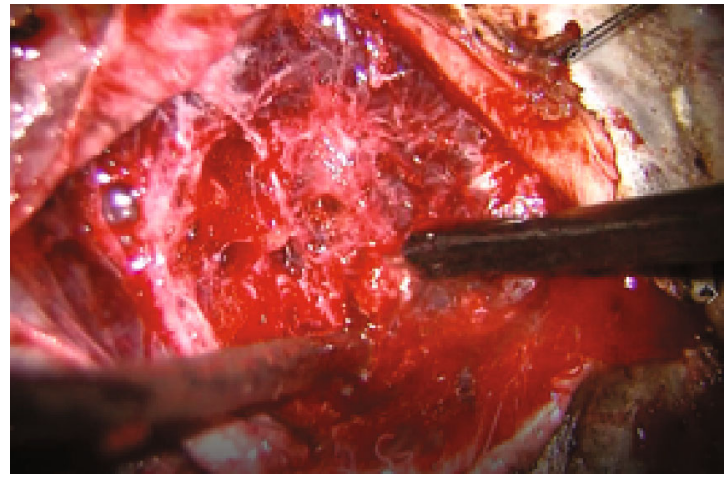

(a)

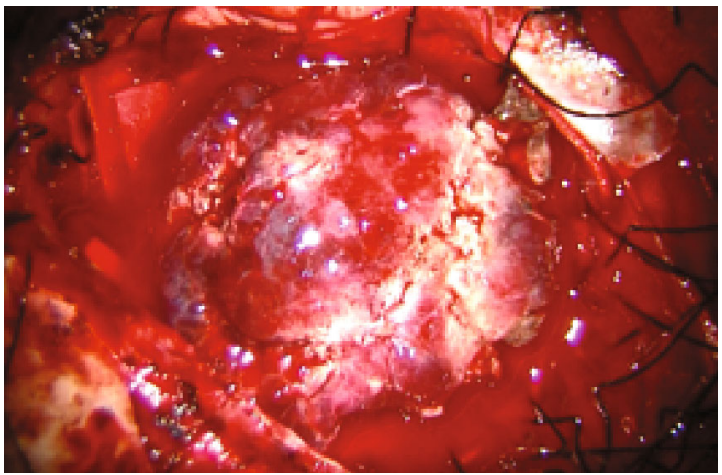

(b)

Figure 4: (a) Intraoperative findings showed that the tumor was hemorrhagic, dark red, and relatively soft and was partially accompanied by fibrous tissue. (b) After the MMA was treated, internal decompression was performed with Sonopet (Stryker Japan KK) to achieve total resection of the tumor, including removal of the infiltrated dura (Simpson grade I).

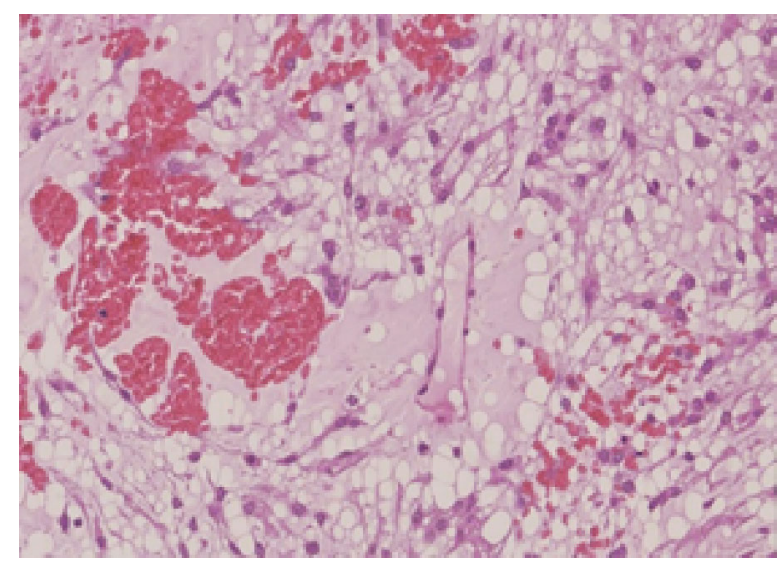

(a)

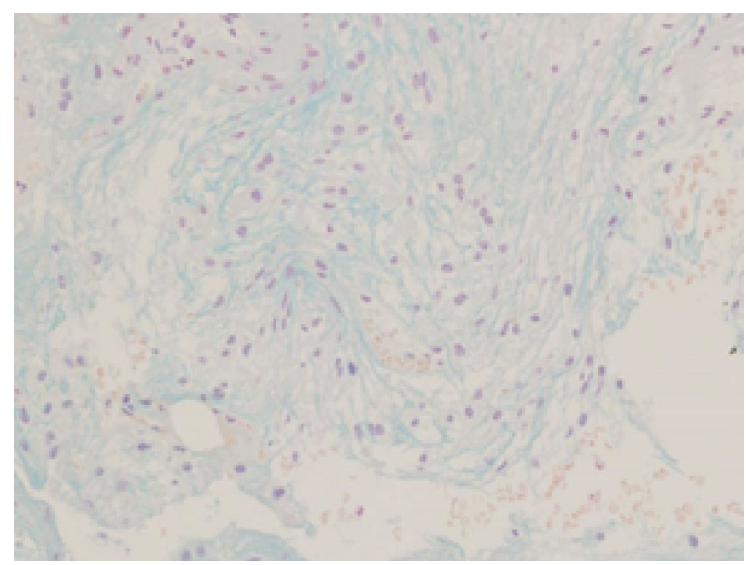

(b)

FIGURE 5: (a) Histopathological analysis showed that fibrous connective tissue containing numerous blood vessels was subfractioned into leaflet-like portions. (b) The areas inside the leaflets were stained with Alcian blue and contained a mucoid matrix. 
Grade II chordoid meningioma is an additional histological subtype of meningioma that commonly requires discrimination. Compared with myxoid meningioma, chordoid meningioma shows a higher grade of dyskaryosis and causes a reticular arrangement with less cytoplasmic vacuolation. In addition, lymphocytes are not observed in myxoid meningioma, while Castleman disease, which causes chordoid meningioma, is characterized by abundant lymphocytes [5].

Myxoid meningioma shows positive immunohistological reactivity for vimentin and EMA and negative reactivity for cytokeratin, HMB45, S-100, actin, desmin, CD117, neurofilaments, and GFAP [6]. A few metaplastic tumors showing positive immunoreactivity for smooth muscle action (SMA) suggest muscular differentiation [7].

We consider that the hemorrhagic, dark red characteristics are due to abundant vascularization. MRI showed significantly hyperintense signals on T2 images. The contrast was successfully enhanced using a Gd-based contrast agent due to the histologically abundant mucoid matrix and vascularization. Our histopathological analysis showed fibrous connective tissue containing numerous blood vessels. In our case, MRI showed very hyperdense signals on T2 images and enhanced Gd-based contrast.

Ki-67 (MIB-1) is used to examine tumor proliferative activity. After nuclear immunostaining, calculating the percentage of cells positive for Ki-67 enables prediction of tumor malignancy, prognosis, and recurrence [8]. In the present case, we were able to perform a total resection with no finding of malignancy. However, cases with a high recurrence, findings of malignancy, and a high score of Ki-67 have been reported; therefore, careful follow-up is necessary.

\section{Conclusion}

We report a rare case of myxoid (metaplastic) meningioma using MRI images and its histopathological features. However, its biological behavior is poorly understood because of the limited number of reported cases. This is a matter that demands full investigation for diagnosis.

\section{Conflicts of Interest}

The authors declare that they have no conflicts of interest.

\section{References}

[1] K. M. Krisht, T. Altay, and W. T. Couldwell, "Myxoid meningioma: a rare metaplastic meningioma variant in a patient presenting with intratumoral hemorrhage," Journal of Neurosurgery, vol. 116, no. 4, pp. 861-865, 2012.

[2] D. D. Bigner, E. R. McLendon, and M. J. Bruner, "Tumors of the cranial, spinal and peripheral nerve sheaths," in Pathology of Tumors of the Nervous System, S. D. Russell and J. L. Rubinstein, Eds., pp. 141-193, Edward Arnold, London, 6th edition, 1998.

[3] H. Tang, H. Sun, H. Chen et al., "Clinicopathological analysis of metaplastic meningioma: report of 15 cases in Huashan Hospital," Chinese Journal of Cancer Research, vol. 25, no. 1, pp. 112-118, 2013.

[4] L. R. Begin, "Intriguing Case: Myxoid meningioma," Ultrastructural Pathology, vol. 14, no. 4, pp. 367-374, 1990.
[5] O. Ozen, A. Sar, B. Atalay, N. Altinörs, and B. Demirhan, "Chordoid meningioma: rare variant of meningioma," Neuropathology, vol. 24, no. 3, pp. 243-247, 2004.

[6] J. Ortiz, M. D. Ludeña, J. Gonçalves, S. Del Carmen, Á. Maillo, and A. Bullon, "Myxoid meningioma: an example of a rare brain Tumour of difficult diagnosis," Open Journal of Pathology, vol. 03, no. 01, pp. 51-53, 2013.

[7] Y.-H. Choi, C.-Y. Choi, C.-H. Lee, H.-W. Koo, and S.-H. Chang, "Metaplastic meningioma overspreading the cerebral convexity," Brain Tumor Research and Treatment, vol. 6, no. 2, pp. 97-100, 2018.

[8] C. F. Begg and R. Garret, "Hemangiopericytoma occurring in the meninges: case report," Cancer, vol. 7, no. 3, pp. 602-606, 1954. 


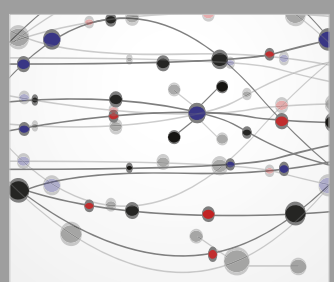

The Scientific World Journal
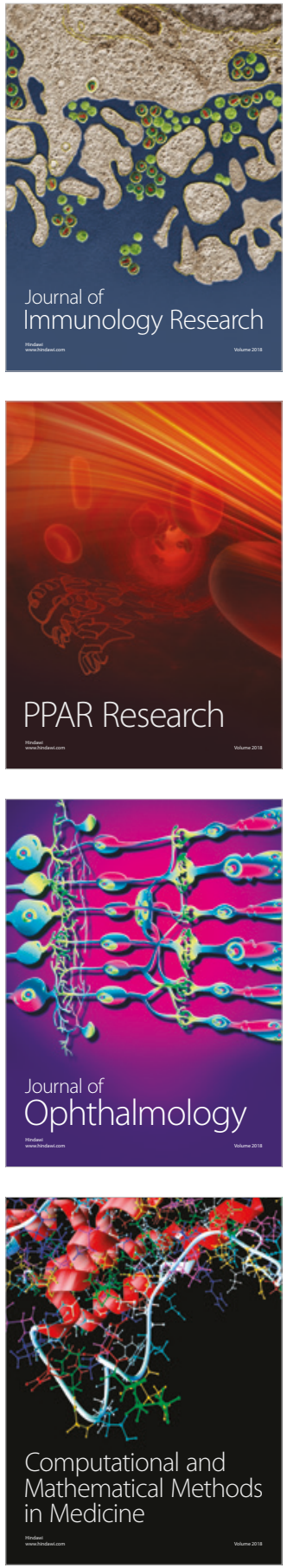

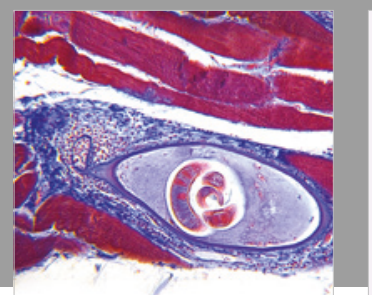

Gastroenterology Research and Practice

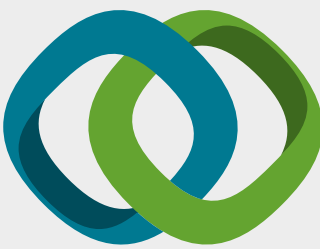

\section{Hindawi}

Submit your manuscripts at

www.hindawi.com
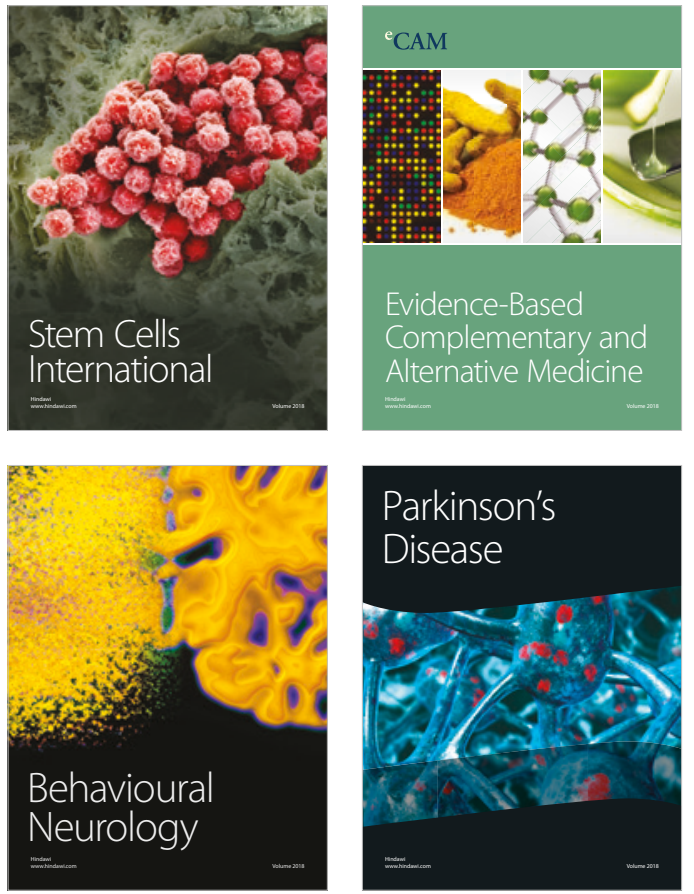

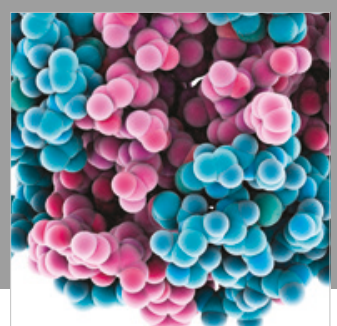

ournal of

Diabetes Research

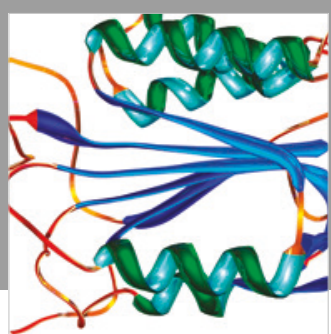

Disease Markers
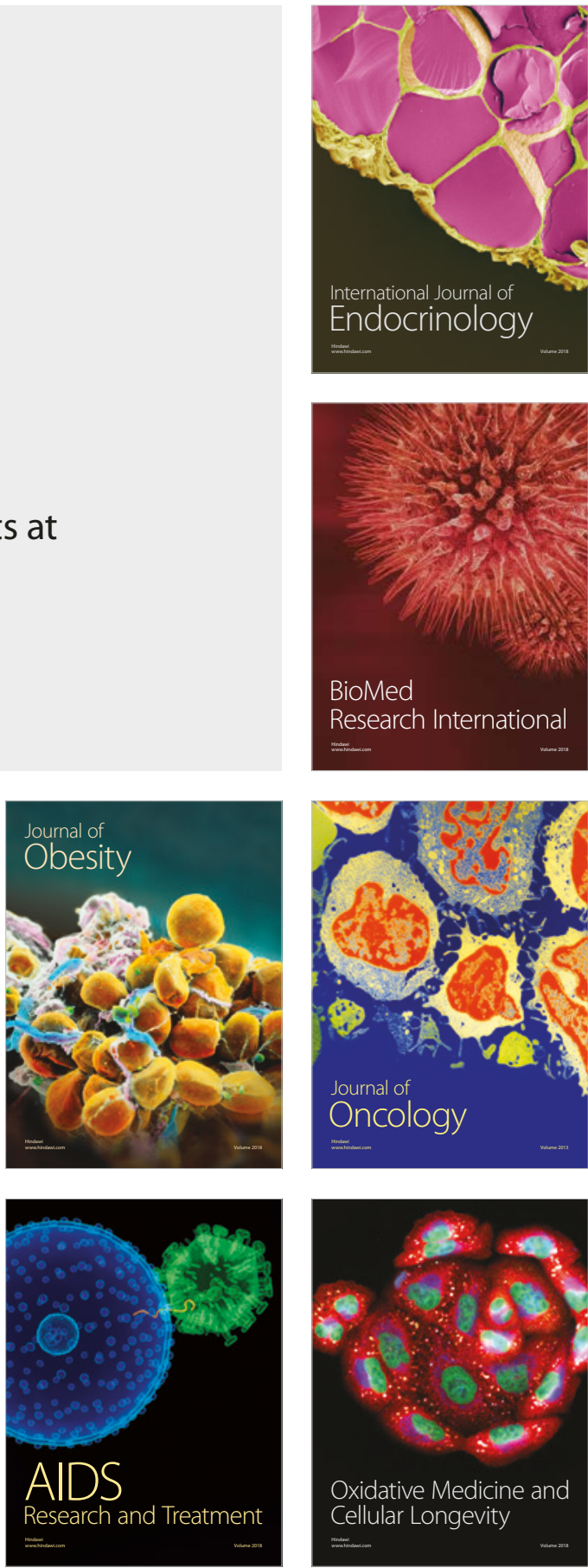